\title{
El conciliador como tercero en la relación contractual: La innovación social en la práctica restaurativa de solución de conflictos
}

The conciliator as a third party in the contractual relationship: Social innovation in restorative conflict resolution practice

O conciliador como terceiro na relação contratual: inovação social na prática de resolução de conflitos restaurativos

Le conciliateur en tant que tiers dans la relation contractuelle: l'innovation sociale dans la pratique de la résolution réparatrice des conflits 合同关系中作为调解人的第三方:在解决冲突的恢复性实践 中的社会创新

Idarmis Knight Soto ${ }^{1}$ y Marla Iris Delgado Knight ${ }^{2}$ Universidad Ciego de Ávila Máximo Gómez Báez - Cuba

Revista Derechos en Acción ISSN 2525-1678/ e-ISSN 2525-1686

Año 5/No 14, Verano 2019-2020 (21 diciembre a 20 marzo), 274-288

DOl: https://doi.org/10.24215/25251678e360

ORCID: https://orcid.org/0000-0003-4713-7488

https://orcid.org/0000-0003-1206-2092

Recibido: 01/12/2019

Aprobado: 01/02/2020

Resumen: El comportamiento entre las partes, basada en su razón jurídica, impone límites al desequilibrio contractual, que en principio se

\footnotetext{
Doctora en Ciencias Jurídicas. Profesora Titular Universidad Ciego de Ávila Máximo Gómez Báez. Consultora Jurídica.

2 MsC. Profesora Asistente. Universidad Ciego de Ávila Máximo Gómez Báez. Notaria
} 
hace previsible en determinadas circunstancias perceptibles, a partir de una comunicación clara influenciada por valores como herramienta para solucionar conflictos con la participación de un tercero, sobre la base de la conciliación entre las partes ${ }^{3}$, centrada en sus necesidades mediante un proceso colaborativo, innovador, y amistoso, con técnicas asertivas, que constituye una nueva forma de entender la defensa jurídica y la justicia, bajo un código deontológico.

Palabras Claves: innovación social, Conciliación, contrato.

Summary: The behavior between the parties, based on their legal reason, imposes limits to the contractual imbalance, which in principle is made foreseeable in certain perceivable circumstances, based on a clear communication influenced by values as a tool to resolve conflicts with the participation of a third party, on the basis of reconciliation between the parties, focused on their needs through a collaborative, innovative, and friendly process, with assertive techniques, which constitutes a new way of understanding legal defense and justice, under a code of ethics.

Keywords: social innovation, Conciliation, contract

Resumo: 0 comportamento entre as partes, com base em sua razão legal, impõe limites ao desequilíbrio contratual, que em princípio é previsível em determinadas circunstâncias perceptíveis, com base em uma comunicação clara, influenciada por valores como ferramenta para solucionar conflitos com a participação de terceiros, com base na reconciliação entre as partes, focada em suas necessidades por meio de um processo colaborativo, inovador e amigável, com técnicas assertivas, que constitui uma nova maneira de entender a defesa legal e a justiça, sob um código de ética.

Palavras-chave: inovação social, conciliação, contrato

Résumé: Le comportement entre les parties, sur la base de leur raison juridique, impose des limites au déséquilibre contractuel, qui est en principe rendu prévisible dans certaines circonstances perceptibles, sur la base d'une communication claire influencée par les valeurs comme outil pour résoudre les conflits avec la participation d'un tiers, sur la base

3 Víd. Art 6-g) de la Resolución 41 del Ministerio de Justicia de fecha 3 de marzo del 2018, publicada en la Gaceta Oficial Extraordinaria № 205 de marzo del 2018 
de la réconciliation entre les parties, centrée sur leurs besoins à travers un processus collaboratif, innovant et convivial, avec des techniques d'affirmation, qui constitue une nouvelle façon d'appréhender la défense juridique et la justice, selon un code d'éthique.

Mots-clés: innovation sociale, conciliation, contrat.

摘要: 当事人之间的行为, 基于其法律原因, 对合同不平衡施加了限 制, 原则上, 在某些可感知的情况下, 合同不平衡是可以预见的, 这 是基于受价值观影响的清晰沟通作为解决与第三方参与冲突的工 具, 在双方之间的和解基础上, 过合作, 创新和友好的流程, 以果断 的技术专注于他们的需求, 这构成了根据道德守则理解法律辩护和 正义的新方式。

关键词: 社会创新, 调解, 合同。

\section{Exordio}

La estructura jurídica de las cláusulas de los contratos es heterogénea configurada típicamente por su contenido, la interpretación como principio rector tiene basamento en la preeminencia de la obediencia debida a las normas imperativas, bajo el principio de la declaración de voluntad de las partes para darle eficacia al acto e imponer un significado que esté íntimamente ligada a la responsabilidad. De ahí, que la información sustancial que se deriva del contrato debe ser oportuna, transparente y de obligatoriedad para las partes.

Cada vez es más creciente la solicitud de tercero o terceros por las partes en litigio para resolver controversias de manera amistosa en la práctica mercantil nacional ${ }^{4}$ e internacional, en sustitución de los litigios judiciales, de cara a construir relaciones económicas armónicas; y es precisamente el objetivo de este artículo, demostrar como la Ley Modelo sobre Conciliación

\footnotetext{
4 Los Estados que deseen incorporar la presente Ley Modelo a su derecho interno para hacerla aplicable a los procedimientos de conciliación tanto nacionales como internacionales tal vez deseen enmendar el texto del modo siguiente: - Suprímase la palabra "internacional" en el párrafo 1 del artículo 1; y — Suprímanse los párrafos 4, 5 y 6 del artículo 1.
} 
Comercial Internacional de la Comisión de las Naciones Unidas para el Derecho Mercantil Internacional ${ }^{5}$, conjuntamente con el Reglamento de Conciliación, recomendado por la Asamblea General en su Resolución 35/52, de 4 de diciembre de 1980, constituyen documentos de tutela jurídico-pública que brindan pautas para lograr acuerdos integrativos que superen las circunstancias conflictuales, aun cuando no exista una ley interna sobre este tema en cuestión.

\section{El conciliador como tercero neutral en el sistema de tutela de las obligaciones contractuales contraídas por las partes}

La "conciliación" se define como todo procedimiento, designado por términos como los de conciliación, mediación o algún otro de sentido equivalente ${ }^{6}$, en el que las partes soliciten a un tercero o terceros ("el conciliador"), que les preste asistencia en su intento por llegar a un arreglo amistoso de una controversia que se derive de una relación contractual $u$ otro tipo de relación jurídica o esté vinculadas a ellas. El conciliador no estará facultado para imponer a las partes una solución de la controversia $^{7}$, propiciará la solución consensual, que permita a los contendientes acatar los puntos de vista de uno u otro, mediante el acuerdo de voluntades con equitativos compromisos, propiciando en el mejor de los casos el fin del conflicto ${ }^{8}$.

La asistencia de un tercero neutral en el procedimiento de conciliación soporta el consenso entre las partes, con la que se limita a facilitar el diálogo, bajo el principio de la confidencialidad,

\footnotetext{
5 Resolución aprobada por la Asamblea General sobre la base del informe de la Sexta Comisión (A/57/562 y Corr.1) 57/18. Ley Modelo sobre Conciliación Comercial Internacional de la Comisión de las Naciones Unidas para el Derecho Mercantil Internacional

6 También se puede conocer como dictamen neutral, miniproceso

7 Víd. Artículo 4 de la Ley Modelo de conciliación de la Ley Modelo de Naciones Unidas

8 Knight Soto I, Delgado Knight M.I, Fournier Duharte, N, (2019) La protección jurídica de los métodos alternativos de solución de conflictos en el ordenamiento jurídico cubano". Realidad y deseo, Congreso de Abogacía 2019 ISBN 978-959-7261-04-9, La Habana, 2019
} 
tres elementos identifican el concepto a decir: la existencia de una controversia, la intención de las partes de llegar a un arreglo amistoso y la participación de un tercero o de terceros independientes e imparciales que ayuden a las partes a llegar a una solución amistosa.

En cambio con secuencias flexibles y sostenidas, en la negociación entre las partes no interviene terceros, se pueden o no adoptar acuerdos a través de las ofertas y contra ofertas en actitudes de competencias, para satisfacer intereses, a través de un proceso para construir acuerdos que perduren en el tiempo a través del diálogo, se trata de la perspicacia competitiva con que se enfrenten los tratos.

Ciertos principios ponen de manifiesto la actitud ética de los conciliadores, los códigos deontológicos lo refuerzan para su ejercicio, la cultura de la conciliación que tomen las partes en las discrepancias que puedan surgir de la ejecución de un contrato, constituye seguridad jurídica y la función que la sociedad demanda de la abogacía, al decir Fajardo Matos y Smith Frrhills 9 , es de triple perspectiva:

1. Reflexionar sobre el valor añadido que un cliente espera que un abogado aporte a la solución de un conflicto mercantil, de ayudar a generar un cambio en la cultura legal, que parta de considerar más los intereses de los clientes para incorporar el diagnóstico de los conflictos y la capacidad estratégica de negociar esos intereses en función y formación del abogado.

2. Deducir de la práctica un método de análisis de los conflictos mercantiles, la sincronía del trabajo del abogado con el interés del cliente exige el análisis previo del conflicto.

3. Definir ciertas pautas para el diseño de estrategia en el uso de los métodos alternativos de solución de conflictos

\footnotetext{
9 FAJARDO MATOS, P Y SMITH FRRHILL S, H, (2015). ¿Para qué sirve un abogado?. EI papel del abogado de Empresa en el siglo XXI, en Revista Anuario de Mediación y Solución de Conflictos. Edit. REUS, S.A, Madrid, pp. 66-67
} 
mercantiles, se trata de entender las razones de filosofía subyacente, que justifican la cooperación de las partes, en vez de enfrentarse, como solución más eficiente de conflictos.

Este tercero imparcial, y especialmente entrenado para este fin, debe dominar eficazmente los factores metodológicos, fundamentalmente por haber adquirido las competencias profesionales esenciales y las habilidades en el manejo de los recursos comunicacionales. (...) como exigencias de las garantías mínimas para el ciudadano que pueda optar por acudir a un proceso de mediación en cuanto a la calidad técnica con la que se pueda desarrollar el proceso de mediación en cuanto a calidad técnica ${ }^{10}$.

El modelo social que se utilice en los procesos de conciliación tiene como premisa el empoderamiento de la persona como ser social, de cara a definir acuerdos justos que satisfagan a las partes.

\subsection{Modalidades del tercero conciliador en el Derecho Colaborativo y la Mediación}

El derecho colaborativo tiene su origen en la conferencia de Pound, en la que el profesor Sander, ofreció la idea de que se ha de posibilitar la forma de resolución más adecuada al conflicto; surgiendo el concepto del tribunal multipuertas, "multi-door courthouse", en la que cada puerta sería una forma de resolución, tal como jurisdicción, mediación, arbitraje, evaluación de expertos, etc. ${ }^{11}$.

En el Derecho colaborativo los abogados realizan una negoción estructurada para satisfacer el interés de los clientes, compartiendo beneficios, desde una perspectiva interdisciplinar, es un proceso voluntario y rápido para construir acuerdos eficaces,

10 ORTUÑO, P., Apuntes críticos sobre la Ley 5/12, de 6 de julio, de mediación Civil y Mercantil, en Revista Anuario de Mediación y Solución de Conflictos, no. 3 ed. REUS. S.A, Madrid, 2013, pp48-49

11 SOleTO MUÑOZ, H, RUÍZ lÓPEZ C., "Elementos esenciales del Derecho Colaborativo", en Anuario de Mediación y Solución de Conflictos, vol. núm. 3. Ed. Reus, S. A., 2016 ISSN: 2340-9681, p 97. 
que de no lograrse pueden acudir las partes en conflictos a la vía jurisdiccional; se ha de apuntar que la representación recae en otros abogados.

Por su parte la mediación se caracteriza por la presencia de un tercero dentro de una estructura singular, que no decide, sin embargo, su oficiosidad en la solución del conflicto posee tres funciones: a) acercamiento de las partes para el análisis flexible del conflicto, b) colaboración para analizar las vías efectivas para dar solución al conflicto c) la adopción de acuerdos que transformen el conflicto como una forma de innovación social a partir de sus intereses.

El tercero conciliador durante el procedimiento debe velar por principios subyacentes como la autodeterminación de las partes, la equidad sustantiva, es decir lograr que el acuerdo, sea justo eficaz, estable e inteligente, así como la justicia del proceso, equidad procedimental ${ }^{12}$.

La equidad sustancial en la conciliación puede determinarse por la remisión a algún reglamento u otra vía, de no existir acuerdo al respecto entre las partes, el conciliador podrá sustanciar el procedimiento conciliatorio del modo que estime adecuado, teniendo en cuenta las circunstancias del caso, los deseos que expresen las partes y la necesidad de lograr un rápido arreglo de la controversia, en cualquier caso, este como tercero procurará dar a las partes un tratamiento equitativo, teniendo en cuenta las circunstancias del caso y en cualquier etapa del procedimiento conciliatorio, podrá presentar propuestas para un arreglo de la controversia, así como llamar a peritos, mediadores, u otros profesionales, se trata de lograr un acuerdo en el cual las partes determinan su contenido.

Por otra parte el perito puede intervenir en el proceso de conciliación, emite un dictamen que no es vinculante, sin

\footnotetext{
12 Cfr. ÁlZATE SÁEZ DE HEREDIA, R., MERINO ORTíZ, C, FERNÁNDEZ VILLANUEVA, I., RUíZ GARCÍA, MA JOSÉ .(2016), "La Intervención en conflictos mediante procesos adaptativos: valores de la mediación e intuicionismo ético" Revista Anuario de Mediación y Solución de Conflictos, $n^{0} 3$ ed. REUS. S.A, Madrid, p. 37.
} 
embargo Redondo Trigo plantea (...) que un informe de ajuste elaborado por un auditor correspondiente, las partes suelen pactar que las posibles diferencias al respecto sean dirimidas definitivamente por otro auditor (que en puridad y por lo que se verá, más parece arbitrador), sometiéndose, de forma firme y vinculante, a la decisión del mismo $(\ldots)^{13}$.

El arbitrador $^{14}$, casi desconocido en nuestra doctrina jurídica, al decir de Diez Picazo ${ }^{15}$, procede del Derecho romano y fue objeto preferente por parte del derecho medieval, no dicta laudo, es el que solventa un extremo no jurídico de una relación jurídica ${ }^{16}$, fija elementos en un contrato, de este no se exige una tasación, sino un parecer, aunque obligatorio, es decir, no se considera arbitraje la intervención de un tercero que no se haga para resolver un conflicto pendiente, sino para completar o integrar una relación jurídica aún no definida totalmente ${ }^{17}$.

Dado que el papel del conciliador se limita a facilitar el diálogo entre las partes, no se necesitan garantías procesales como las que existen en el arbitraje, por ejemplo, la prohibición de que el conciliador se reúna sólo con una de las partes o la obligación incondicional de revelar a una parte toda la información recibida de la otra. Se considera, por tanto, de importancia crucial la flexibilidad que permiten los procedimientos de conciliación y la posibilidad de adaptar el proceso a las circunstancias de cada caso y a los deseos de las partes.

\footnotetext{
13 REDONDO TRIGO, F.(2007), La impugnación de la determinación del precio por un tercero en los contratos de compraventa de acciones y la sentencia del Tribunal Supremo de 1.9.2006, RCDI, noviembre-diciembre, p. 27766ss en ROGEL VIDE, C., Arbitradores, Peritos y Árbitros Revista Anuario de Mediación y Solución de Conflictos, $n^{0} 3$ ed. REUS. S.A, Madrid, 2013, pp. 72-73

14 Víd. Artículo 1447 del Código Civil Español,

15 DIEZ PICAZO, L. (1957), El arbitrio de tercero en los negocios jurídicos, Bosch, Barcelona, p. 170.

16 Víd. Sentencia de 29 de noviembre de 2010 del Tribunal Supremo Español

17 Víd. Art 2.II de la Ley Española sobre Régimen Jurídico de los Arbitrajes de Derecho Privado de 22 de diciembre de 1953
} 
Es dable aclarar que el tercero conciliador, salvo acuerdo en contrario de las partes ${ }^{18}$, no es árbitro, ni asesor o representante de una parte cuando participe en un procedimiento conciliatorio, ni en otra controversia que surja a raíz del mismo contrato o relación jurídica o de cualquier contrato o relación jurídica conexos, aunque sí podrán disponer la prestación de asistencia administrativa por una institución o persona idónea. Esta decisión es supletoria y fortalece la confianza hacia el conciliador, con la finalidad de facilitar el desarrollo del procedimiento conciliatorio, la exégesis del acuerdo se realizará de buena fe, generando un cambio en la situación de controversia, con resultado vinculante y susceptible de ejecución.

El éxito de la conciliación es un acuerdo de transacción que pone fin al procedimiento previa consulta con las partes ${ }^{19}$, sin embargo puede resultar lo contrario, el abandono una vez comenzado éste, siempre que estuviera implícito en la conducta de las partes, por ejemplo, en una opinión negativa de una de las partes sobre las perspectivas de conciliación, o en el hecho de que una de las partes se negara a consultar o reunirse con el conciliador cuando se la invitaba a hacerlo, en estos casos queda expedita la vía judicial.

Si las partes llegan a un acuerdo por el que se resuelva la controversia, dicho acuerdo será vinculante y susceptible de ejecución, este encuadre preliminar del tema permite llegar a la vía ejecutoria sumaria, se reconoce en algunos ordenamientos en el supuesto en que las partes y sus asesores letrados firmen un arreglo de transacción que contenga una declaración, también cuando un arreglo haya sido certificado por notario o refrendado por un juez.

\footnotetext{
18 (A/CN.9/514, párrafo 71).Víd. Observaciones sobre el artículo 12 de la Ley Modelo sobre Conciliación

19 (A/CN.9/514, párrafo 69)
} 


\section{La construcción de acuerdos, como innovación social. Una mirada al Derecho Comparado}

En el Derecho Comparado podemos encontrar diversos ejemplos, así citaremos en la legislación de Bermudas se dispone, que si las partes en un acuerdo de arbitraje que prevea el nombramiento de un conciliador resuelven sus diferencias y firman un acuerdo en que se detallen las condiciones del arreglo, el acuerdo de transacción será tratado, a efectos de su ejecución, como un laudo en un acuerdo de arbitraje y, con el consentimiento del tribunal o de un juez del tribunal, podrá ejecutarse del mismo modo que un fallo o un mandamiento al mismo efecto y, si se autoriza expresamente, se podrá dictar un fallo en los términos del acuerdo ${ }^{20}$.

En la India todo acuerdo de transacción que hayan firmado las partes es definitivo y vinculante para éstas y para las personas cuyos derechos estén supeditados a los de aquéllas, y tendrá la misma validez y el mismo efecto que un laudo arbitral ${ }^{21}$.

En Alemania, la solución amistosa de litigios en el procedimiento civil y mercantil, el Código de procedimiento Civil, establece que el juez puede remitir a las partes a un juez de calidad, el cual puede utilizar métodos alternativos de solución de conflictos, como la mediación, conciliación y el arbitraje y con la aprobación de las partes puede acudir a conversaciones individuales; las negociaciones se realizan en presencia de los abogados de las partes y su objetivo es finalizar el litigio ${ }^{22}$.

No obstante, en algunos ordenamientos la ejecutoriedad de un acuerdo de transacción concertado durante el procedimiento conciliatorio dependerá de que el acuerdo se haya concertado en el marco de un arbitraje o de un acuerdo de arbitraje. Por ejemplo, en la Región Administrativa Especial de Hong Kong

\footnotetext{
20 Bermudas, Arbitration Act, 1986.

21 India, Arbitration and Conciliation Ordinance, 1996, artículos 73 y 74

22 Víd. Art. 278, pár.5 ZPO
} 
(China), cuando un procedimiento de conciliación prospera y las partes celebran un acuerdo de transacción por escrito (antes o durante un procedimiento de arbitraje), el Tribunal de Primera Instancia podrá ejecutar ese acuerdo como si fuera un laudo, siempre y cuando haya sido concertado por las partes en un acuerdo de arbitraje $\mathrm{e}^{23}$.

En Cuba se avanza en el reconocimiento jurídico de normas jurídicas sobre las prácticas restaurativas de solución de controversias $^{24}$, la Constitución ${ }^{25}$, recién aprobada, apunta a ello, precedida por principios reconocidos que se identifican por la participación voluntaria, responsabilidad propia, imparcialidad o neutralidad, flexibilidad, información y confidencialidad, que se inscriben para diseñar respuestas delimitadas ante las necesidades personales, con intervención colaborativa.

El Reglamento de Mediación de la Corte Cubana de Arbitraje Comercial Internacional, establece que el Acuerdo o Acuerdos de Mediación tendrán carácter definitivo y fuerza vinculante para las partes, las cuales se obligan a su cumplimiento en los términos acordados. En caso de su incumplimiento, su ejecución forzosa puede ser solicitada por la parte que se considere con derecho a ello, ante los tribunales ordinarios competentes, de conformidad con lo establecido en la Ley ${ }^{26}$.

23 En la sección 2C de la Arbitration Ordinance (Cap. 341, enmendada con efecto a partir del 27 de junio de 1997) se dispone: Si las partes en un procedimiento de arbitraje logran dirimir su controversia y concertar un acuerdo por escrito que contenga los términos del arreglo ("el acuerdo de transacción"), ese acuerdo de transacción, a los efectos de su ejecutoriedad, será tratado como un laudo en un acuerdo de arbitraje y, con el consentimiento del tribunal 0 de uno de sus magistrados, podrá ser ejecutado de la misma forma que un fallo 0 un mandamiento a ese efecto y, si se autoriza expresamente, se dictará un fallo en los términos del acuerdo.

24 Resolución publicada en la Gaceta Oficial № 57 Extraordinaria del 3 de Octubre de2018. Reglamento de Mediación de la Corte Cubana de Arbitraje Comercial Internacional, de fecha 16 de abril del 2018.

25 Artículo 93 de la Constitución de la República del 10 de abril del 2019.

26 Víd artículo 37 de la Resolución 9 de fecha 16 de abril del 2018, publicada en la gaceta Extraordinaria de la República de Cuba № 57, 2018. 
Cuando los acuerdos finales concretados en proceso de mediación internacional hayan de ejecutarse en otro Estado, resulta necesario el cumplimiento de los requisitos establecidos en los Convenios internacionales ratificados por el Estado cubano en la materia objeto de la mediación cuando ello resulte procedente o aplicable, y de conformidad con lo que al respecto se establezca en el foro de ejecución. 2. La resolución judicial extranjera contentiva de los acuerdos finales concretados en proceso de mediación internacional no podrá ser reconocida y ejecutada en territorio cubano, cuando resulte manifiestamente contraria al orden público de la República de $\mathrm{Cuba}^{27}$.

Resulta interesante advertir que la conciliación preprocesal también reconocida en la legislación patria, permite que juez el actúe como conciliador, es imparcial, constituye otro mecanismo de acercamiento complementario para la solución de conflictos.

La sociedad del conocimiento hace necesaria una aproximación más flexible y holística a los problemas, desde la conciliación ante nuevas demandas, acercando a las partes en la solución de controversias, bajo las garantías de voluntariedad, igualdad, transparencia y confidencialidad como solución amigable ante los incumplimientos de obligaciones contractuales.

\section{Epílogo}

Las nuevas realidades apuntan hacia la utilización de la conciliación como método alternativo de solución de conflictos, el conciliador como tercero imparcial, gestiona la búsqueda de soluciones satisfactorias a la controversia en cuestión, a través de los intereses subyacentes que justifican la participación de las partes, en este sentido la adopción de acuerdos constituye una innovación social, toda vez que contribuye a actualizar, proyectar o reformular las cláusulas del contrato en ejecución, desde una cultura de paz.

\footnotetext{
27 Víd. Artículo 18 de la Resolución 9 de fecha 16 de abril del 2018, publicada en la gaceta Extraordinaria de la República de Cuba № 57, 2018
} 


\section{Bibliografía}

ALZATE SÁEZ DE HEREDIA, R., Merino Ortíz, C., Fernández Villanueva, I., Ruíz García, Ma José .(2015), ”La Intervención en conflictos mediante procesos adaptativos: valores de la mediación e intuicionismo ético" Revista Anuario de Mediación y Solución de Conflictos, $\mathrm{n}^{\circ} 3$ ed. REUS. S.A, Madrid, 2015

DIEZ-PICAZO, L.(1957), El arbitrio de tercero en los negocios jurídicos., Bosch, Barcelona.

FAJARDO MATOS, P Y SMITH FRRHILL S, H, (2015).¿Para qué sirve un abogado?. El papel del abogado de Empresa en el siglo XXI, en Revista Anuario de Mediación y Solución de Conflictos. Edit. REUS, S.A, Madrid

FERNÁNDEZ BULTÉ, J.(2004), Teoría del Derecho, Ed. Félix Varela, La Habana.

KNIGHT SOTO I, DELGADO KNIGHT M.I, Y FOURNIER DUHARTE, N. (2019), La protección jurídica de los métodos alternativos de solución de conflictos en el ordenamiento jurídico cubano". Realidad y deseo, Congreso de Abogacía 2019 ISBN 978-9597261-04-9, La Habana, 2019

LARROUMET, CH.(2006) "La unificación del derecho de las obligaciones en Europa”, Revista de Derecho Privado, nueva época, año V, n. ${ }^{\circ}$ 13-14, París, Universidad Pantheón-Assas (París II), 69-80, recuperado en:http://www.juridicas.unam.mx/publica/ librev/rev/derpriv/cont/13/dtr/dtr4.pdf

ORTUÑO, P.(2013), Apuntes críticos sobre la Ley 5/12, de 6 de julio, de mediación Civil y Mercantil, en Revista Anuario de Mediación y Solución de Conflictos, $\mathrm{n}^{\circ} 3$ ed. REUS. S.A, Madrid.

REDONDO TRIGO, F.(2007), La impugnación de la determinación del precio por un tercero en los contratos de compraventa de acciones y la sentencia del Tribunal Supremo de 1.9.2006, RCDI, noviembre-diciembre, p. 27766ss en ROGEL VIDE, C., Arbitradores, Peritos y Árbitros Revista Anuario de Mediación y Solución de Conflictos, $\mathrm{n}^{\circ} 3$ ed. REUS. S.A, Madrid, 2013.

SOLETO MUÑOZ, H, RUÍZ LÓPEZ C., "Elementos esenciales del Derecho Colaborativo", en Anuario de Mediación y Solución de Conflictos, vol. núm. 3. Ed. Reus, S. A., 2016 ISSN: 2340-968 
TOHARIA CORTES, J.J.(2006), "Las profesiones Jurídicas, una Aproximación Sociológica”, en Diez Picazo L.M., (Coordinador), $E l$ oficio del Jurista, Siglo XXI, Madrid.

ZENNER, A., LEBEAU, J.P. (2010), La Loi Relative a la continueité des entreprises a l'épreuve de sa premiere pratique, monografía publicada como $\mathrm{n}^{\circ} 6$ de Les Dossiers du Journal des Tribunaux, Bruselas.

\section{Legislación}

Constitución de la República de Cuba, de fecha10 de abril de 2019, Editora política ISBN 978-959-01-1065-8, La Habana, 2019

Decreto-Ley No 304/2012 "De la Contratación Económica" del Consejo de Estado de fecha 1 de noviembre del 2012 publicado en la Gaceta Oficial de la República de Cuba Ordinaria en fecha 27 de diciembre del 2012

Decreto No 310/2012 "De los Tipos de Contratos", del Consejo de Ministros de fecha 17 de noviembre del 2012 publicado en la Gaceta Oficial de la República de Cuba Ordinaria en fecha 27 de diciembre del 2012

Ley Modelo sobre Conciliación Comercial Internacional de la Comisión de las Naciones Unidas para el Derecho Mercantil Internacional, aprobada mediante Resolución aprobada por la Asamblea General sobre la base del informe de la Sexta Comisión (A/57/562 y Corr.1) 57/18.

Reglamento de Conciliación, recomendado por la Asamblea General en su Resolución 35/52, de 4 de diciembre de 1980

Principios UNIDROIT sobre los Contratos Comerciales Internacionales 2010, International Institute for the Unification of Private Law, Unidroit, Roma, disponible: [http://www.unidroit.org/ spanish/principles/contracts/principles2010/blackletter2010spanish.pdf.

Resolución 9 de fecha 16 de abril del 2018, publicada en la Gaceta Extraordinaria de la República de Cuba No 57, 2018 A/CN.9/514, párrafo 69 


\section{Jurisprudencia}

Sentencia del Tribunal Supremo, Sala primera, de lo Civil, 2 de marzo de 2011. El deber de secreto que alcanza el mediador y las partes se refiere "a las informaciones confidenciales", pero no al acuerdo libremente adoptado y referido a las consecuencias (...), " Revista Anuario de Mediación y Solución de Conflictos, $\mathrm{n}^{\circ} 3$ ed. REUS. S.A, Madrid, 2015 